\title{
Separation of Magnetic Particles Using an Array of Magnets - A Model of a Separation Device for Malaria-Infected Blood Cells
}

\author{
Surasak Kasetsirikul, Werayut Srituravanich, \\ Prapruddee Piyaviriyakul, ${ }^{1}$ and Alongkorn Pimpin* \\ Department of Mechanical Engineering, Faculty of Engineering, Chulalongkorn University, \\ Bangkok 10330, Thailand \\ 'Department of Physiology, Faculty of Veterinary Medicine, Chulalongkorn University, Bangkok 10330, Thailand
}

(Received August 1, 2016; accepted January 4, 2017)

Keywords: malaria, cell separation, magnetic microparticles, magnet array, microfluidics, mathematical model

Our ultimate goal is to design, fabricate, and test a new platform for malaria diagnosis using an array of magnets located parallel to a straight microchannel. The principle of the diagnosis is to attract malaria-infected blood cells using a nonuniform magnetic field induced by the array of magnets, while healthy blood cells are not affected and move along the flow direction. To achieve the goal, a mathematical model for predicting blood-cell motion was developed and validated using magnetic particles. In the experiments, trajectories of magnetic particles were captured using a photographic technique as the particles moved inside the system. The study has revealed that the trajectories of the magnetic particles obtained from both computational and experimental results were in a good agreement, and the mean deviation between them was around $18 \%$ for both 5 and $10 \mu \mathrm{m}$ magnetic particles. In addition, the simulation results for malaria-infected mouse blood cells suggested that at the distance of $400 \mu \mathrm{m}$ from the magnet array, the infected blood cells could move laterally toward the magnet array at distances around $35.2,26.9,21.8$, and $18.3 \mu \mathrm{m}$ within a $3 \mathrm{~cm}$ downstream distance at flow rates of $0.18,0.23,0.28$, and $0.33 \mu \mathrm{L} / \mathrm{min}$, respectively.

\section{Introduction}

Malaria has become a serious healthcare problem worldwide, especially in tropical countries. $^{(1-3)}$ Asymptomatic malaria infection has been accepted as a major difficulty for malaria control and elimination. Therefore, a quick and accurate diagnosis using blood tests is needed for separating infected patients from a community so that the disease does not spread and can be kept under control. However, a conventional diagnosis, microscopic analysis using blood smears, needs highly skilled technicians and laboratory facilities; therefore, medical services for malaria diagnosis in outbreak areas, e.g., border regions between countries with few resources, are usually insufficient. Recently, the rapid diagnostic test (RDT) taking advantage of lateral flow in nitrocellulose membranes embedded with specific proteins was considered to be an effective technique to control malaria due to its ease of use without the need of a skilled technician.

*Corresponding author: e-mail: alongkorn.p@chula.ac.th http://dx.doi.org/10.18494/SAM.2017.1442 
However, this technique is prone to bio-degrade under improper storage conditions and to give false-positive detection owing to the existence of malaria antigens in blood circulation even after the malaria parasites had been eliminated. Another technique which is the most efficient to date is polymerase chain reaction (PCR)-based analysis. Although the technique has a high sensitivity and specificity, it depends upon a primer sequence, and the cost per test is still relatively high compared with others. Therefore, alternative techniques are needed to overcome the problem. One of them is a microfluidic device that is cheap, requires less diagnosis time, uses small amounts of samples, and does not need a skilled technician. ${ }^{(4-10)}$

Recently, hemozoin, produced by a malaria parasite invading red blood cells, is being considered as an alternative biomarker for malaria diagnosis. Many research studies demonstrated that malaria-infected red blood cells (iRBCs) show magnetic properties distinct from those of healthy red blood cells (hRBCs), as shown in Table 1, and many groups have already utilized this difference for detecting or separating iRBCs in a label-free manner on a high gradient of a magnetic field inside the microfluidic device. ${ }^{(4-8)}$

However, the current design has some limitations, such as being a batch process, having low precision, or causing severe damage to iRBCs. One important issue to be resolved is the significant reduction in the magnitude of the magnetic force with distance from the magnet poles. Zimmerman et al. demonstrated the new platform utilizing the gap between two different poles of magnets to increase the concentration of infected blood cells, but its operation requires multiple steps. ${ }^{(4)}$ The microfluidic channel combined with a ferromagnetic wire in a microscale, on the other hand, is difficult to operate as well as requiring a highly precise fabrication. ${ }^{(5)}$

To tackle the problem, we employed an array of magnets, as an array can locally generate a high gradient of magnetic field as well as a magnetic force that are comparable to a single magnet pole. Although the magnitude of magnetic force from a single magnet is slightly higher, the capability to extend a high-force region along a microchannel using the array of magnets is necessary. To design a new system, we proposed a mathematical model to predict the motion of iRBCs in the magnetic field induced by a magnet array. The system consists of a straight microfluidic channel with the magnet array placed parallel to the channel. The trajectories of objects moving inside the system are monitored using a video camera, and the validation of the mathematical model is performed by comparing the computational and experimental results using magnetic particles under the same conditions.

\section{Materials and Methods}

\subsection{Computational study and validation}

Figure 1 shows the configuration of our computational system. It consists of a straight microchannel and an array of permanent magnets. The coordinate system is defined by giving the $x$-axis as in the flow direction, the $y$-axis toward the ceiling of the channel, and the $z$-axis toward the

Table 1

Relative magnetic susceptibilities of each component in this study. ${ }^{(2,3)}$

\begin{tabular}{lccc}
\hline Relative magnetic susceptibility & Magnetic particles & iRBCs & hRBCs \\
\hline$\Delta \chi$ & 0.2601 & $1.8 \times 10^{-6}$ & $0.01 \times 10^{-6}$ \\
\hline
\end{tabular}

Remark: $\Delta \chi$ is calculated from the difference between the magnetic susceptibilities of medium and objects. 


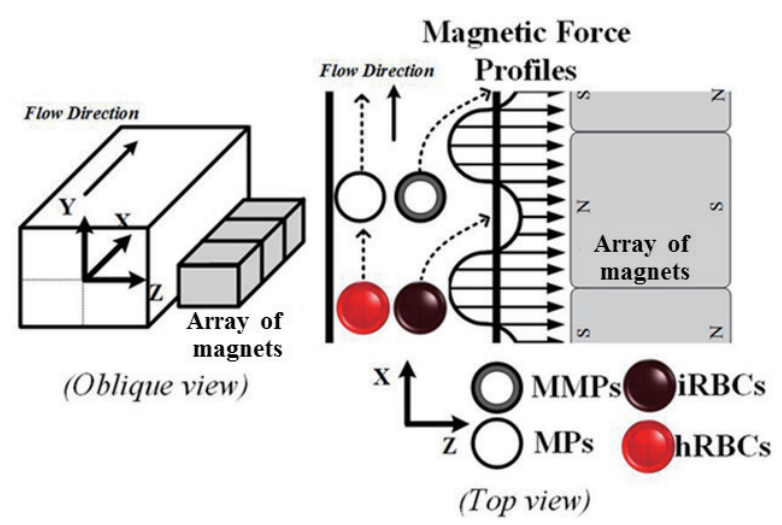

(a)

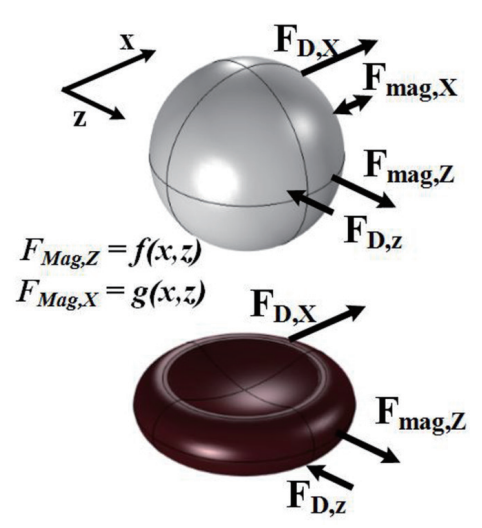

(b)

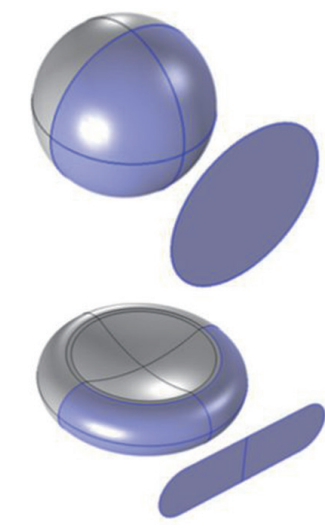

(c)

Fig. 1. (Color online) Magnetic-particle and erythrocyte model: (a) computational domain, (b) forces acting in each direction, and (c) surface area of the objects exposed to fluid flow.

side wall. With this system, the iRBCs are attracted by the magnetic force and move laterally across streamlines toward the magnet array. The magnitude of magnetic force due to the magnetophoresis principle can be described as

$$
F_{m}=\frac{2}{3} \pi \mu_{0} \Delta \chi a^{3} \nabla|\vec{H}|^{2}
$$

where $F_{m}$ is the magnitude of magnetic force, $\mu_{0}$ is the permeability of free space, $\Delta \chi$ is the relative susceptibility, $a$ is the diameter of targeted objects, and $H$ is the magnetic field norm. In accordance with Eq. (1), the magnitude of the magnetic force is proportional to the gradient of magnetic field which can be enhanced by appropriate system design.

In this study, a magnet array is proposed since it makes the fabrication process and system arrangement simple resulting in ease of operation and possibility of observing the phenomena inside the microfluidic microchannel. The conceptual principle and computational domain are shown in Fig. 1(a). Two object models, i.e., iRBCs and magnetic micro-particles, are shown in Fig. 1(b).

The forces acting on objects suspended in the flow are composed of drag force, pressuregradient force, added-mass force, and Basset force, as well as an externally active force, according to the Basset-Boussinesq-Oseen equation. In this case, the externally active force is the magnetic force expressed in Eq. (1). The Basset and added-mass forces are related to the acceleration of both flowing fluid and particles. Because of the slow flow speed as well as low acceleration, the effect of Basset and added-mass forces could be neglected in this study.(7) In addition, to simplify the mathematical model, the effect of pressure-gradient force is also ignored. In Refs. 11 and 12, there are some arguments as to whether it is necessary to include the pressure-gradient force in the equation of motion.

Therefore, only drag and magnetic forces are included in the mathematical model similar to that in Ref. 7, and the force diagrams in both axes are summarized in Fig. 1(b). The equations of motion in the $x$ - and $z$-directions of the suspended objects are

$$
F_{m, x}+6 \pi \mu_{f} a\left(v_{f}-v_{o b j, x}\right)=\rho_{o b j} \forall \frac{d^{2} x}{d t^{2}},
$$




$$
F_{m, z}-6 \pi \mu_{f} a v_{o b j, z}=\rho_{o b j} \forall \frac{d^{2} z}{d t^{2}}
$$

where $F_{m, x}$ and $F_{m, z}$ are defined as magnetic forces acting on a suspended object, $\mu_{f}$ is the viscosity of fluid, $a$ is the radius of the object, $\rho_{o b j}$ is the density of the object, $\forall$ is the volume of the object, $\frac{d^{2} x}{d t^{2}}$ and $\frac{d^{2} z}{d t^{2}}$ are the accelerations of objects in streamwise and lateral directions, and $v_{f}$ and $v_{o b j}$ are the velocities of fluid and objects, respectively. On the basis of the motion in the vertical direction (y-direction), it is assumed that the suspended objects are already settled on the bottom surface of the microchannel before the beginning of the computation domain. The distance from the beginning to the point where the objects settle down was previously approximated, and this distance was found to be relatively short compared with the length of the straight microchannel. Therefore, in this study our model considers as two-dimensional laminar flow by neglecting motion in $y$-direction similar to those in Refs. 13-16. In addition, the magnetic force in the streamwise direction is neglected in the simulation since its value is a few orders of magnitude smaller than that of the drag force in the same direction.

To validate the mathematical model, the examination of the motion of magnetic particles was carried out numerically and experimentally. In the simulation, the magnetic particle was modeled as a sphere. The magnitude of drag force was calculated relative to the surface area of sphere exposed to the flow, which was different from the erythrocyte model as shown in Fig. 1(c). However, the blood cells were still considered as rigid objects without changes in orientation. With the assumptions of the erythrocyte model, the surface area of erythrocytes exposed to the flow was a constant value in the mathematical model.

The calculation of the magnetic force began with the employment of COMSOL Multiphysics software to compute the magnetic field distribution as shown in Fig. 2(a). After that, using Eq.

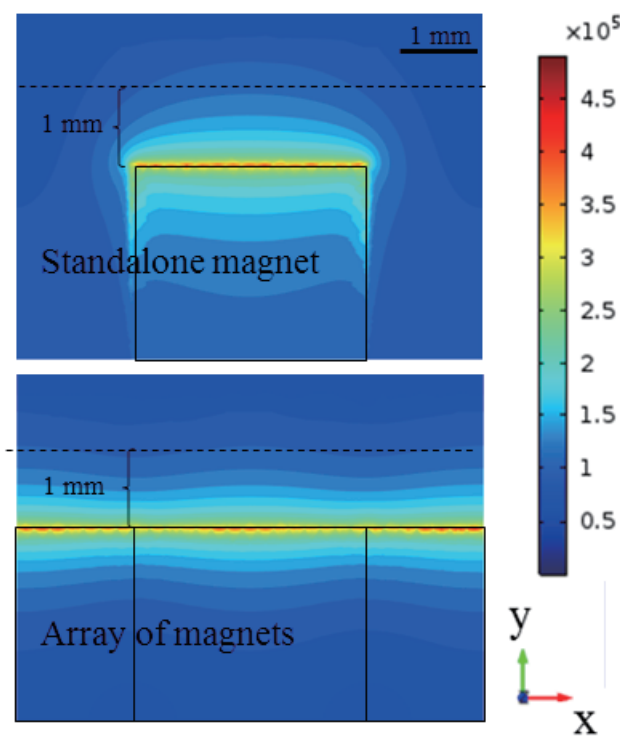

(a)

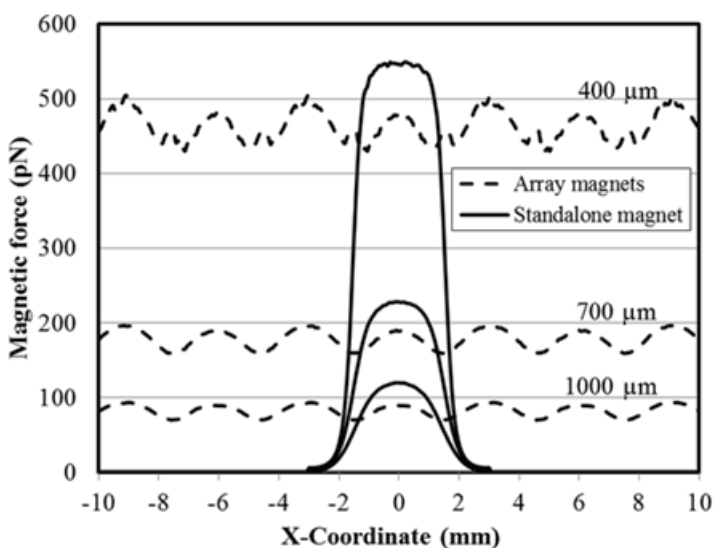

(b)

Fig. 2. (Color online) Magnetic field distribution induced by the magnet array and single magnet and force distribution exerted on $5 \mu \mathrm{m}$ magnetic particles at distances of 400, 700, and $1000 \mu \mathrm{m}$ from the magnet. 
(1) and the constant parameters in Table 2, the magnitude of magnetic force on each single object at different locations in the microfluidic microchannel was approximated as shown in Fig. 2(b). Consequently, the motion of magnetic particles was numerically calculated using Eqs. (2) and (3) with a finite difference method employing MATLAB programming. The next position of the magnetic particles from the given initial location was calculated by defining a small time step $(h$ $=10^{-7} \mathrm{~s}$ ), which was reiterated to obtain a trajectory of the magnetic particles until they reached the side wall. Furthermore, some assumptions, such as no interaction between cells, little effect of viscosity, no particle (or cell) rolling, and small surface-tension force, were adopted in this study.

\subsection{Fabrication process and experiments}

The polydimethylsiloxane (PDMS) device consists of three inlets and two outlets with a 3-cmlong straight microchannel. The microchannel is $500 \mu \mathrm{m}$ wide and $100 \mu \mathrm{m}$ thick. The permanent magnets, each with a magnetic strength of $0.42 \mathrm{~T}$ and dimensions of $3 \times 3 \times 5 \mathrm{~mm}^{3}$, are placed in a line array on the side of the microchannel. According to the process flow presented in Fig. 3(a), the conventional soft-lithography method was employed to fabricate the microchannel using a casting mold made from a computer numerical control (CNC)-processed acrylic plate. PDMS was bonded to a glass slide using $\mathrm{O}_{2}$ plasma, and tubes were later connected. The fabricated device is shown in Fig. 3(b).

Table 2

Constant parameters in the computational study.

\begin{tabular}{lc}
\hline Parameters & Value \\
\hline Viscosity of water at $25^{\circ} \mathrm{C}$ & $8.93 \times 10^{-4} \mathrm{Ns} / \mathrm{m}^{2}$ \\
Density of water at $25^{\circ} \mathrm{C}$ & $997 \mathrm{~kg} / \mathrm{m}^{3}$ \\
Density of blood cells & $1125 \mathrm{~kg} / \mathrm{m}^{3}$ \\
Density of coated magnetic polystyrene (size $5 \mu \mathrm{m})$ & $1530 \mathrm{~kg} / \mathrm{m}^{3}$ (Sigma Aldrich) \\
Density of coated magnetic polystyrene (size $10 \mu \mathrm{m})$ & $1710 \mathrm{~kg} / \mathrm{m}^{3}$ (Sigma Aldrich) \\
Magnetic susceptibility & $0.26($ Sigma Aldrich) \\
Nominal diameter of magnetic beads (size $5 \mu \mathrm{m})$ & $4.8 \mu \mathrm{m}$ \\
Nominal diameter of magnetic beads (size $10 \mu \mathrm{m})$ & $9.6 \mu \mathrm{m}$ \\
\hline
\end{tabular}

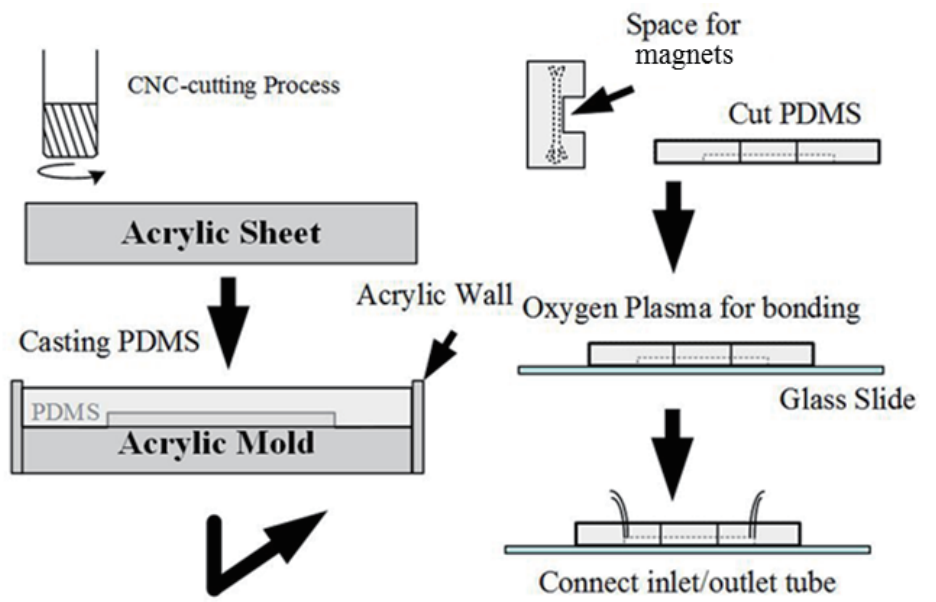

(a)

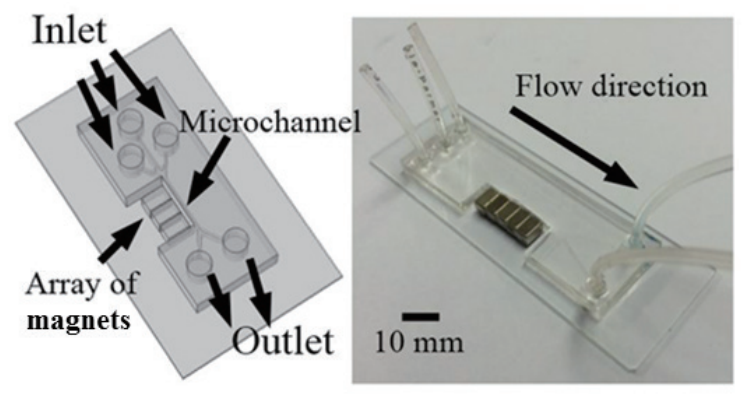

(b)

Fig. 3. Fabrication of the PDMS microchannel: (a) process flow and (b) schematic of the chip (drawn) and the actual chip used in the experiment. 
The experiments to validate the mathematical model were performed using two types of magnetic particles, i.e., 5 and $10 \mu \mathrm{m}$ diameter magnet-coated beads (Sigma Aldrich). For the $5 \mu \mathrm{m}$ magnetic particles, the experiments were conducted at a concentration of $1.5 \times 10^{6}$ beads $/ \mathrm{mL}$ at a flow rate of $2 \mu \mathrm{L} / \mathrm{min}$. For the $10 \mu \mathrm{m}$ magnetic particles, experiments were conducted at $7.4 \times 10^{5}$ beads $/ \mathrm{mL}$ and $20 \mu \mathrm{L} / \mathrm{min}$.

Figure 4 is a schematic diagram of the experimental setup that consists of two syringe pumps for driving the samples into the system and an optical microscope with a 15 fps camera for observing the trajectory of particles. An example of a captured image is shown in Fig. 5(a). It was clearly seen that the magnetic particles were laterally attracted towards the magnet array,

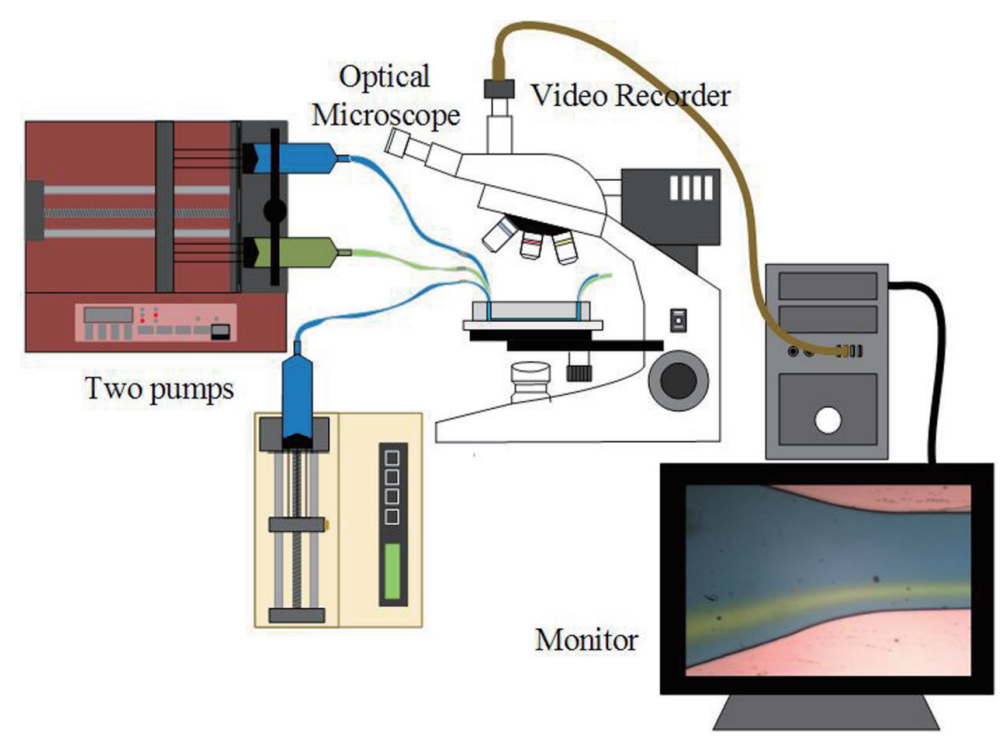

Fig. 4. (Color online) Schematic diagram of the experimental setup.

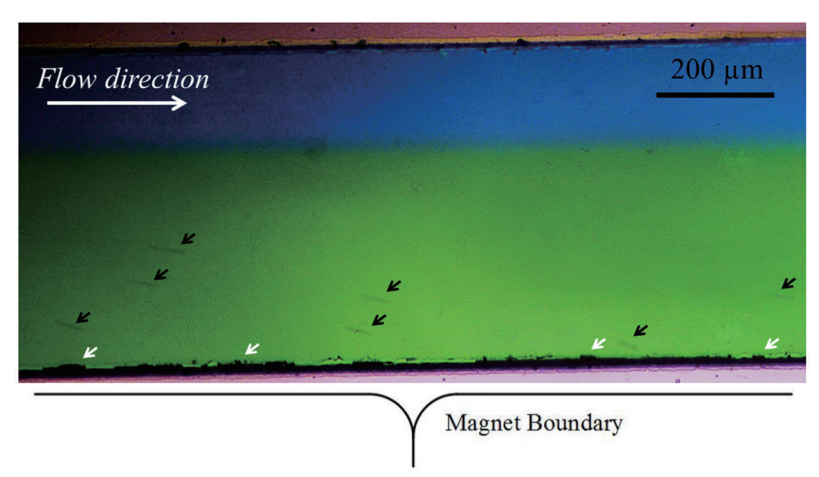

(a)

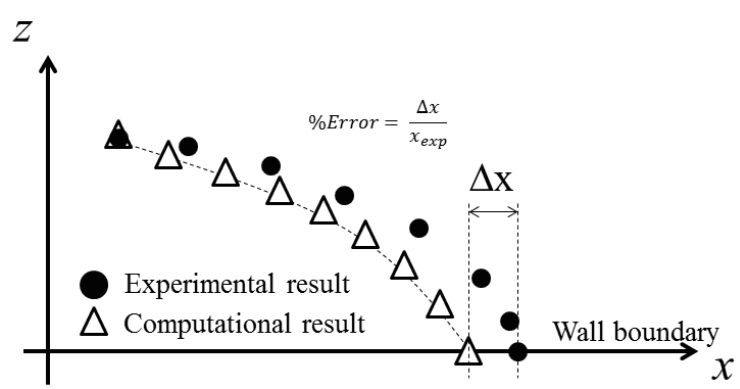

(b)

Fig. 5. (Color online) Image obtained in the experiment to examine the particle trajectory: (a) photograph obtained using the optical microscope and (b) concept for approximating the deviation of trajectory between two studies. Sample (upper) and buffer (lower) streams were distinguished using different colors of stain. Black arrows point to the moving $5 \mu \mathrm{m}$ particles, and white arrows point to the $5 \mu \mathrm{m}$ particles stuck on the wall under a flow rate of $2 \mu \mathrm{L} / \mathrm{min}$. 
and, later on, some magnetic particles were stuck to the side wall. By comparing two consecutive images, the distance moved by one particle between the time intervals could be approximated. The measurement of the distance moved between each position was repeated three times with an uncertainty of $1-2 \mu \mathrm{m}$. The experiment to examine the particle trajectory under the same conditions was conducted three times to verify the consistency of the device as well as the experimental setup.

To validate the mathematical model, the trajectory data collected from the experiment were compared with those from the mathematical model at the same initial position. The results from both techniques were compared and the difference in the distance along the $x$-axis on the channel wall, where the magnetic particles collided, was obtained. This difference was defined as the error of the mathematical model as shown in Fig. 5(b).

In the end, the malaria parasite, $P$. berghei, in mouse blood samples was employed in the system. Hemozoin in this plasmodium was reported to be similar to that found in humans. ${ }^{(17,18)}$ After drawing a blood sample from a malaria-infected mouse, the sample was treated with Nycodenz solution to separate infected cells in the Schizont stage, which contains a large amount of hemozoin compared with other stages. After that, the prepared blood sample was injected into the system at an initial distance from the side wall of $100 \mu \mathrm{m}$, corresponding to $400 \mu \mathrm{m}$ from the magnet array, at a flow rate of $0.4 \mu \mathrm{L} / \mathrm{min}$ with the concentration of the infected cells counted using a hemocytometer (ISOLAB) being around $1.75 \times 10^{10}$ cells $/ \mathrm{mL}$. During the experiments, the motion of the suspended blood cells was observed at two locations inside the system. The first location (Area I) was at the beginning of the straight channel, and the second location (Area II) was at the end of the straight channel as shown in Fig. 6.

\section{Results}

Figure 7 shows a comparison of the trajectories from the computational and experimental results for 5 and $10 \mu \mathrm{m}$ magnetic particles, respectively. The graphs show that the trajectory was slightly turned toward the side wall until a distance of around $50 \mu \mathrm{m}$ from the wall. After that, the trajectory turned rapidly to the direction almost normal to the side wall in both cases. This might be the effect of the magnetic field gradient that strongly influenced within the location of 150-200 $\mu \mathrm{m}$ away from the magnet array.

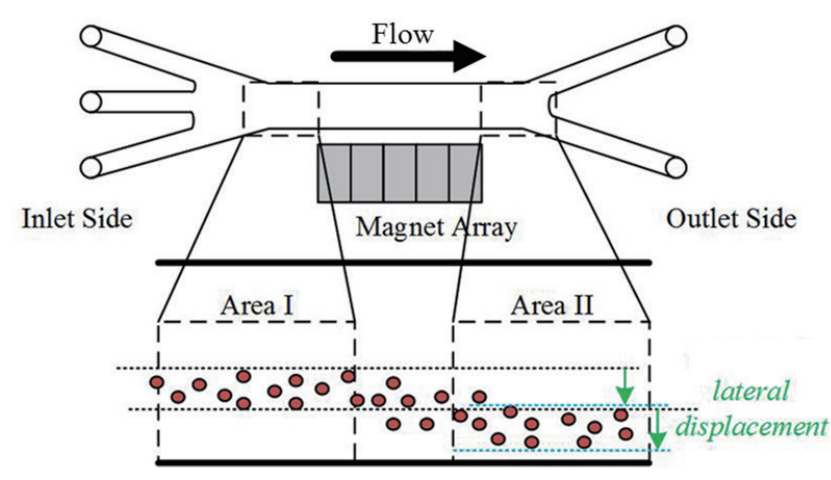

Fig. 6. (Color online) Two observation windows in real blood-cell experiments. 


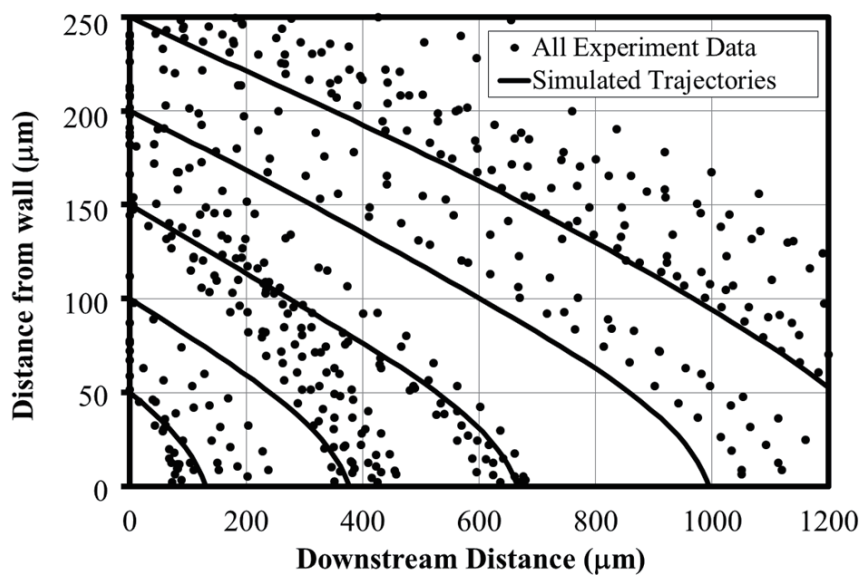

(a)

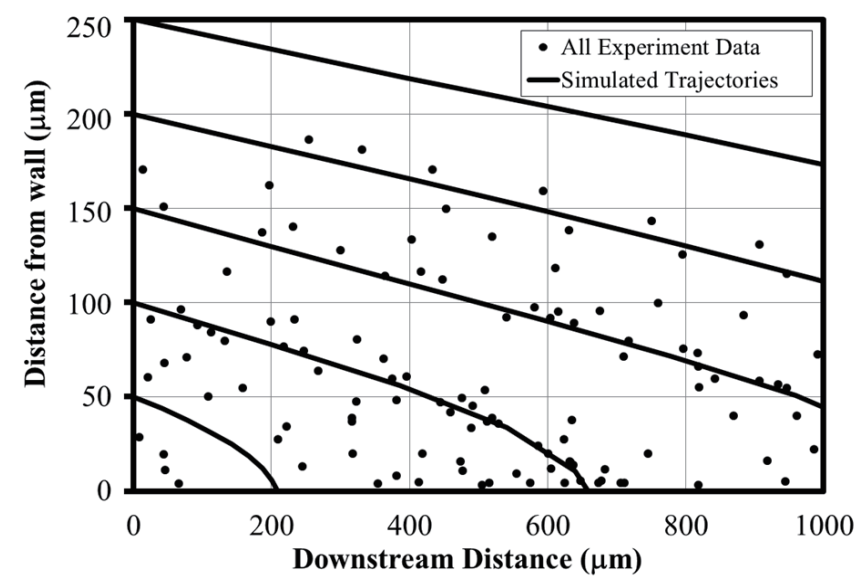

(b)

Fig. 7. Experimental trajectories of magnetic particles compared with those from the mathematical model: (a) $5-\mu \mathrm{m}$ from 473 data sets and (b) $10-\mu \mathrm{m}$ from 139 data sets.

In both cases, the trajectories of the particles from the experiments turned towards the side wall slowly compared with those in the mathematical model. This observation is probably due to an exposure to a lower magnitude of magnetic force that is a result of overrating the magnetic field or the magnetic properties of the magnetic particles in the mathematical model. A comparison of the result of the two studies indicates that our mathematical model had an average deviation of around $18 \%$ for both particle sizes.

From the experiments, the standard deviation in the measured distance when constructing trajectories was approximately $1 \%$, which is much lower than the deviation between the computational and experimental studies. Thus, the cause of the deviation between the two studies could be the difference between the constant values of the physical properties or the boundary conditions employed in the two studies. For example, in the experiments, there was a nonuniform distance between the magnet array and the channel wall that might lead to a fluctuation in the magnetic force of 6.1 and $9.0 \%$ along the channel length on average for 5 and $10 \mu \mathrm{m}$ magnetic beads, respectively. Additionally, some physical properties of the magnet array and magnetic particles (as shown in Tables 1 and 2) could have some uncertainties resulting from their heterogeneity.

For the malaria-infected blood-cell model, the trajectories of infected blood cells were calculated, and the results are shown in Fig. 8. The results suggested that, when the initial position of the infected cells was at a distance of $100 \mu \mathrm{m}$ from the side wall, the distances through which infected cells were laterally attracted toward the side of the wall in the presence of the magnet array were around $35.2,26.9,21.8$, and $18.3 \mu \mathrm{m}$ within $3 \mathrm{~cm}$ downstream of the microchannel at flow rates of $0.18,0.23,0.28$, and $0.33 \mu \mathrm{L} / \mathrm{min}$, respectively. With such a short distance observed under a microscope, the lateral motion of mouse blood cells was difficult to observe due to the diffusion of the bloodstream at the low speed of the flow. Despite of that, the wider spread of the bloodcell stream at a flow rate of $0.4 \mu \mathrm{L} / \mathrm{min}$, as shown in Fig. 9, suggested that the mouse blood cells were drawn by the magnetic force toward the magnet array. In order to attract cells with a longer lateral distance, the spacing between the magnet array and the focused blood-cell stream should be reduced. 


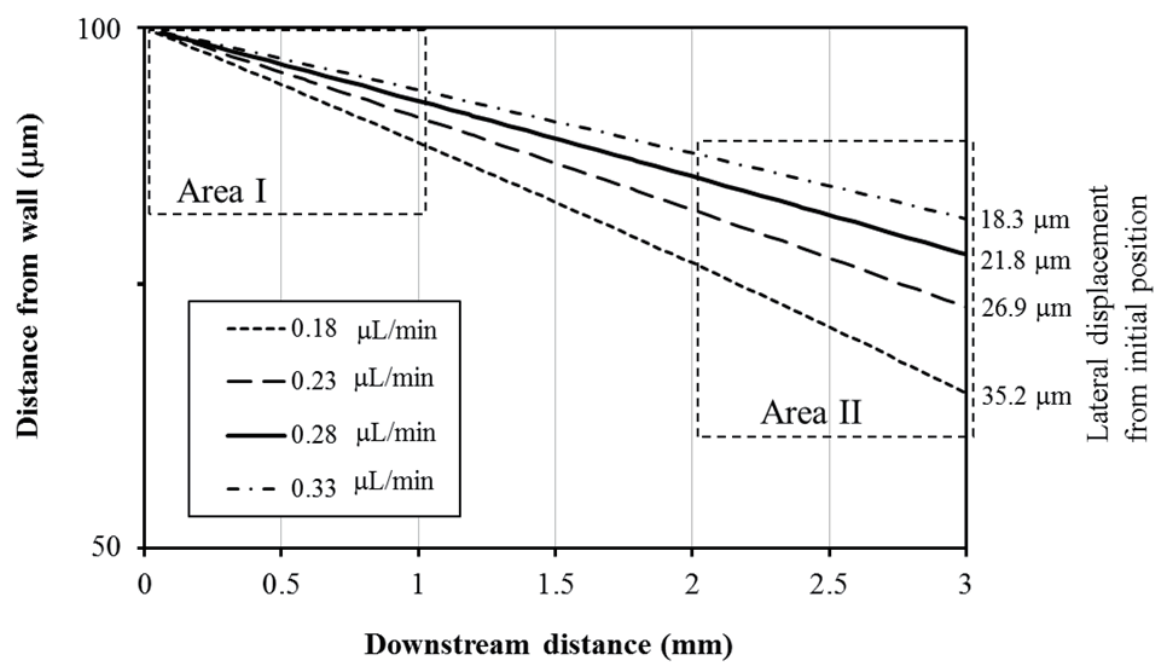

Fig. 8. Simulated trajectories of malaria-infected erythrocytes under various flow rates at the focused initial distance of $400 \mu \mathrm{m}$ from the magnet array, or $100 \mu \mathrm{m}$ from the side wall.

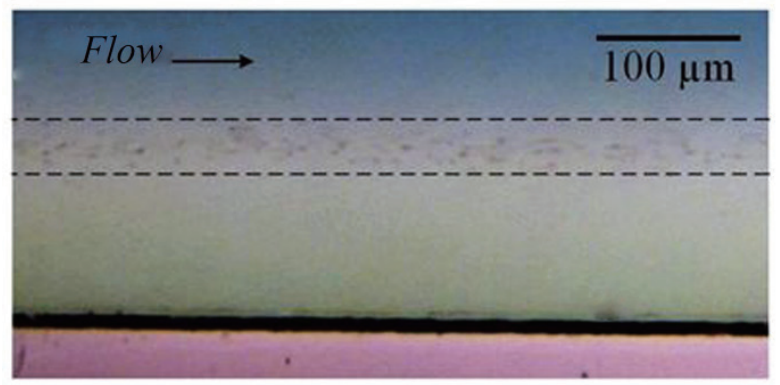

Area I

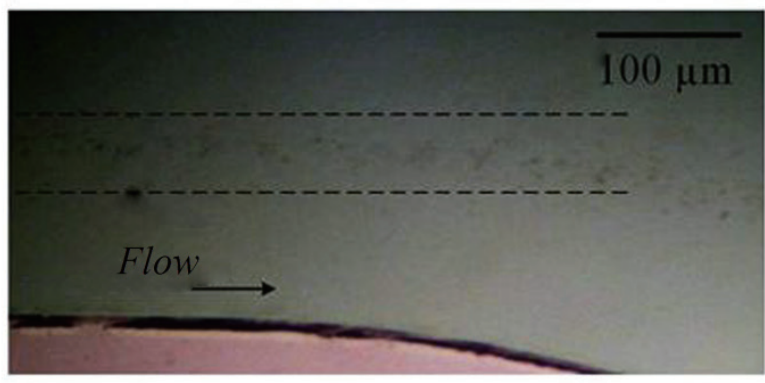

Area II

Fig. 9. (Color online) Images from the mouse-blood experiments in the presence of the magnet array. Dashed lines represent the extent of a spreading of the blood-cell stream at a flow rate of $0.4 \mu \mathrm{L} / \mathrm{min}$.

\section{Conclusions}

The purpose of this study was to develop and validate a mathematical model to predict the motion of malaria-infected blood cells owing to the magnetic force in a nonuniform magnetic field. The lateral trajectory of the blood cells toward the magnet array placed on the side wall of microchannel was computed using COMSOL Multiphysics and MATLAB software. To validate the model, experiments under the same conditions were carried out in a microfluidic channel to examine the trajectories of magnetic particles. Two sizes of magnetic particles, i.e., 5 and $10 \mu \mathrm{m}$, were employed at flow rates of 2 and $20 \mu \mathrm{L} / \mathrm{min}$, respectively. Then, the trajectories in two studies were compared, and the results demonstrated that there was an approximately $18 \%$ deviation between the two studies for both sizes of magnetic particles. This deviation could be a result of the difference of the physical properties and boundary conditions in the simulation and the real 
experiments as well as the imprecision of the fabrication process. In the study of infected blood cells, the mathematical model suggested that the infected blood cells would move laterally by around $35.2 \mu \mathrm{m}$ within $3 \mathrm{~cm}$ downstream at a distance of $400 \mu \mathrm{m}$ from the magnet array under a flow rate of $0.18 \mu \mathrm{L} / \mathrm{min}$. Nonetheless, in the experiments, this short lateral motion of the infected blood cells including the effect of diffusion under a low flow rate made observation difficult. The results suggested that the infected blood cells would be drawn by the magnetic force from the magnet array.

\section{Acknowledgements}

This work received financial support from Chulalongkorn University through the Chulalongkorn Academic Advancement into Its 2nd Century Project (Smart Medical Device).

\section{References}

1 World Health Organization (WHO): World Malaria Report 2014 (2014).

2 R. E. Coleman, J. Sattabongkot, S. Promstaporm, N. Maneechai, B. Tippayachai, A. Kengluecha, N. Rachapaew, G. Zollner, R. S. Miller, J. A. Vaughan, K. Thimasarn, and B. Khuntirat: Malar. J. 14 (2006) 121.

3 S. Kasetsirikul, J. Buranapong, W. Srituravanich, M. Kaewthamasorn, and A. Pimpin: Malar. J. 15 (2016) 358.

4 P. A. Zimmerman, M. Thomson, H. Fujioka, W.E. Collins, and M. Zborowski: Am. J. Trop. Med. Hyg. 4 (2006) 74.

5 J. Nam, H. Huang, H. Lim, C. Lim, and S. Shin: Anal. Chem. 85 (2013) 7316.

6 T. F. Kong, W. Ye, W. K. How, Marcos, P. R. Preiser, N. T. Nguyen, and J. Han: Sci. Rep. 5 (2015) 1.

7 J. Kim, M. Massoudi, J. F. Antaki, and A. Gandini: Appl. Math. Comput. 218 (2012) 6841.

8 T. J. Egan: J. Inorg. Biochem. 102 (2008) 1288.

9 P. Gascoyne, J. Satayavivad, and M. Ruchirawat: Acta Tropica 89 (2004) 357.

10 P. Rolfe: Sens. Mater. 24 (2012) 275.

11 S. L. Loo: Phys. Fluids 18 (1975) 263.

12 S. L. Loo: Phys. Fluids 19 (1976) 757.

13 S. Vyas and G. Friedman: J. Magn. Magn. Mater. 423 (2017) 301.

14 M. Berger, J. Castelino, R. Huang, M. Shah, and R. H. Austin: Electrophoresis 22 (2001) 3883.

15 D. W. Inglis, R. Riehn, R. H. Austin, and J. C. Sturm: Appl. Phys. Lett. 85 (2004) 5093.

16 J. D. Adams, U. Kim, and H. T. Soh: Proc. Natl. Acad. Sci. USA 17 (2008) 2008.

17 T. Hanschied, T. Calvalho, and M. P. Grobusch: Trends Parasitol. 32 (2016) 94.

18 C. J. Janse, J. Ramesar, and A. P. Waters: Nat. Protoc. 1 (2006) 346.

\section{About the Authors}

Surasak Kasetsirikul obtained his bachelor degree in mechanical engineering in 2014 from Chulalongkorn University, Thailand. He is currently a master's student in the Department of Mechanical Engineering, Chulalongkorn University. His research interests include microfluidics technology and computational simulation of various physical phenomena.

Werayut Srituravanich received his Ph.D. in mechanical engineering from UCLA in 2007. Currently, he is working as an assistant professor at the Department of Mechanical Engineering, Chulalongkorn University, Thailand. His research interests include microsystems, nanomaterials, and nanomedicine. He is the author and co-author of numerous publications including articles in Nature Nanotechnology, Nature Materials, and Nano-Letters. 
Prapruddee Piyaviriyakul received his Ph.D. in applied marine bioscience from Tokyo University of Marine Science and Technology in 2006. Currently, he is in the Unit of Biochemistry, Department of Physiology, Faculty of Veterinary Science, Chulalongkorn University, Thailand. His research interests include canine mast cell tumors, parasitic disease, and microfluidics technology.

Alongkorn Pimpin obtained his bachelor and master's degrees in mechanical engineering in 1997 and 2000, respectively, from Chulalongkorn University, Thailand. In 2005, he received his doctoral degree in mechanical engineering from The University of Tokyo. After that, he returned to Thailand and has been working as an assistant professor in the Department of Mechanical Engineering, Chulalongkorn University, since 2012. His research interests include micro-actuators, lab-on-chip, and biomedical engineering technology. 